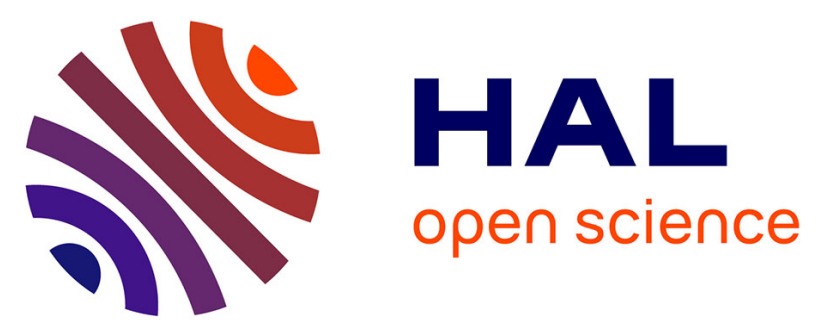

\title{
Comparison of MRI-derived vs. traditional estimations of fatty acid composition from MR spectroscopy signals
}

Angeline Nemeth, Berenice Segrestin, Benjamin Leporq, Amandine Coum, Giulio Gambarota, Kévin Seyssel, Martine Laville, Olivier Beuf, Hélène Ratiney

\section{To cite this version:}

Angeline Nemeth, Berenice Segrestin, Benjamin Leporq, Amandine Coum, Giulio Gambarota, et al.. Comparison of MRI-derived vs. traditional estimations of fatty acid composition from MR spectroscopy signals. NMR in Biomedicine, 2018, 31 (9), pp.e3991. 10.1002/nbm.3991 • hal-01861324

\section{HAL Id: hal-01861324 https://hal.science/hal-01861324}

Submitted on 24 Aug 2018

HAL is a multi-disciplinary open access archive for the deposit and dissemination of scientific research documents, whether they are published or not. The documents may come from teaching and research institutions in France or abroad, or from public or private research centers.
L'archive ouverte pluridisciplinaire HAL, est destinée au dépôt et à la diffusion de documents scientifiques de niveau recherche, publiés ou non, émanant des établissements d'enseignement et de recherche français ou étrangers, des laboratoires publics ou privés. 


\section{Comparison of MRI-derived vs. traditional}

2 estimations of fatty acid composition from

\section{MR spectroscopy signals}

4

5

6

7

8

9

10

Angeline Nemeth ${ }^{1}$, Bérénice Segrestin ${ }^{2}$, Benjamin Leporq ${ }^{1}$, Amandine Coum ${ }^{3,4}$, Giulio Gambarota ${ }^{3,4}$, Kevin Seyssel ${ }^{5}$, Martine Laville ${ }^{2}$, Olivier Beuf ${ }^{1}$ and Hélène Ratiney ${ }^{1 *}$

${ }^{1}$ Univ Lyon, INSA-Lyon, Université Claude Bernard Lyon 1, UJM-Saint Etienne, CNRS, Inserm, CREATIS UMR 5220, U1206, F69621, Lyon, France, ${ }^{2}$ Centre de Recherche en Nutrition Humaine Rhône-Alpes (CRNH-RA), Centre Hospitalier Lyon Sud, Pierre-Bénite, Lyon, France ${ }^{3}$ INSERM, UMR 1099, Rennes, France, ${ }^{4}$ Univ Rennes 1, LTSI, Rennes, France, ${ }^{5}$ Department of Physiology, Faculty of Biology and Medicine, University of Lausanne, Lausanne, Switzerland,

Keywords (8): fatty acid composition, in vivo MR spectroscopy, fitting approach, adipose tissue, parameter estimation, ill-conditioned problem

\section{Correspondence to:}

Hélène Ratiney, Univ Lyon, INSA-Lyon, Université Claude Bernard Lyon 1, UJM-Saint Etienne, CNRS, Inserm, CREATIS UMR 5220, U1206, F69621, Lyon, France

Email: helene.ratiney@creatis.insa-lyon.fr 
25 Abstract

Introduction - The composition of fatty acids in the body is gaining increasing interest, and can be followed up noninvasively by quantitative MR spectroscopy (MRS). However, current MRS quantification methods have been shown to provide different quantitative results in terms of lipid signals, with possible varying outcomes for a given biological examination. Quantitative MR imaging using multigradient echo sequence (MGE-MRI) has recently been added to MRS approaches. In contrast, these methods fit the undersampled MR temporal signal with a simplified model function (expressing the triglyceride (TG) spectrum with only three TG parameters), specific implementations and prior knowledge. In this study, an adaptation of a MGE-MRI method to MRS lipid quantification is proposed.

Methods - Several versions of the method - with time data fully or undersampled, including or excluding the spectral peak T2 knowledge in the fitting - were compared theoretically and on Monte Carlo studies to a time-domain peak-fitting approach. Robustness, repeatability and accuracy were also inspected on in vitro oil acquisitions and test-retest in vivo subcutaneous adipose tissue (SAT) acquisitions, adding results from the reference LCModel method.

Results - On simulations, the proposed method provided TG parameter estimates with the smallest variability but with a possible bias, which was mitigated by fitting on undersampled data and considering peak T2s. For in vitro measurements, estimates for all approaches were correlated with theoretical values and the best concordance was found for the usual MRS method (LCModel and peak fitting). Limited in vivo test-retest variability was found (4.1\% for PUFAindx, $0.6 \%$ for MUFAindx, 3.6\% for SFAindx), as for LCModel (7.6\% for PUFAindx, 7.8\% for MUFAindx, 3.0\% for SFAindx).

Conclusion - This study shows that fitting the three TG parameters directly on MRS data is one valuable solution to circumvent the poor conditioning of the MRS quantification problem. 


\section{Abbreviations used}

52

53

54

55

56

57

58

59

60

61

62

63

64

65

66
- FA, fatty acid;

- SFA, saturated fatty acid;

- MUFA, monounsaturated fatty acid;

- PUFA, polyunsaturated fatty acid;ndb, number of double bonds;

- $\quad n m i d b$, number of methylene-interrupted double bonds;

- SFAindx, proportion of saturated fatty acid estimated by $n d b$ and $n m i d b$;

- MUFAindx, proportion of monounsaturated fatty acid estimated by $n d b$ and $n m i d b$;

- PUFAindx, proportion of polyunsaturated fatty acid estimated by $n d b$ and $n m i d b$;

- $\quad \mathrm{CL}$, chain length;

- $\quad$ SAT, subcutaneous adipose tissue;

- SD, standard deviation

- $\mathrm{CV}$, coefficient of variation;

- CRLB, Cramer Rao Lower Bound.

- PDFF, proton density fat fraction 


\section{Introduction}

Interest in fat quantification has grown in recent years. In particular, the fat composition in the body could play a role in various inter-related pathologies or disorders such as obesity, inflammation, insulin resistance and cardiovascular disease risk. It is therefore worth developing accurate and robust tools to measure and follow the fatty acid composition in the body noninvasively. In this respect, Magnetic Resonance Spectroscopy (MRS) has been shown to be a quantitative, noninvasive technique that can assess this fat composition ${ }^{1-6}$. The spectral content of the ${ }^{1} \mathrm{H}$ lipid spectrum, e.g., acquired in adipose tissue ${ }^{7,8}$, bone marrow ${ }^{2,8}$ or liver $\mathrm{fat}^{6,9}$, is related to the types of triglycerides (saturation, unsaturation, polyunsaturation). The different types of triglycerides are obtained from the quantification of the area under the curve (in the spectral domain) or equivalently from the signal amplitude (in the time domain) of the different proton components. In this context, Hamilton et al. ${ }^{6}$ introduced the notion of the number of double bonds $(n d b)$, the number of double bonds separated by a single $\mathrm{CH}_{2}$ (nmidb, the number of methylene-interrupted double bonds) and chain length $(C L)$. These triglyceride (TG) parameter values link the amplitude of each resonating peak. With these relations, indexes related to the percentage of saturated fatty acids (SFAindx), monounsaturated fatty acids (MUFAindx), and polyunsaturated fatty acids (PUFAindx) can be deduced. Until now, proton peaks have been quantified by quantification algorithms such as AMARES (Advanced Method for Accurate Robust and Efficient Spectral) ${ }^{10}$, LCModel (Linear Combination of Model spectra) ${ }^{11,12}$, Automated Quantification of Short Echo-time MRS (AQSES)-Lineshape ${ }^{13,14}$, and quantitation based on QUantum ESTimation (QUEST) ${ }^{15}$. However, as has been recently demonstrated $\mathrm{in}^{16}$, the quantification results can greatly differ from one quantification algorithm to another, which could influence the statistical outcome of a biological investigation. Moreover, next to these dedicated MRS quantification approaches, quantitative MR imaging using multigradient echo sequence (MGEMRI) has recently been proposed ${ }^{17-21}$. These MGE-MRI approaches proved their ability to quantify TG fatty acid composition, from far fewer spectroscopic data points (i.e., 1024 points compared to 8-15 echoes). To make this possible, this approach relies on more assumptions than in the MRS quantification approaches as well as directly estimated $n d b$, nmidb and $C L$ based on a simplified model. Then the question arises as to whether MRI approaches can be adapted to the MR spectroscopic signal and how their statistical performance would compare with the usual MRS quantification methods.

In this paper, we therefore investigated several quantification strategies - derived from the MGEMRI approach or employing the usual MRS approaches - in particular the quantitative analysis of the spectroscopic adipose tissue lipid signal. In the continuation of the work reported in ${ }^{16}$ analyzing the results of different quantification methods, we analyzed possible sources of error and discrepancies 
between different quantification approaches. The aim of this study was to evaluate and reduce the uncertainties and errors of estimates. After Monte Carlo studies examining several fitting implementations, the quantification results obtained with different quantification models, including results from the reference LCModel method, were analyzed for in vitro oil acquisitions and in vivo spectroscopy subcutaneous adipose tissue (SAT) acquisitions. These analyses resulted in a number of practical solutions and considerations for robust lipid composition assessment using MR spectroscopic signals.

\section{Materials and methods}

\section{Model function and quantification strategies}

Most of the current MRS quantification methods are based on a nonlinear least squares analysis that fits the acquired spectroscopic data points with a parametric model function. Note that in the following "quantification" means "relative quantification" and the goal, for all the methods studied, is to determine from a MR spectroscopy signal, the relative contribution of PUFAindx, MUFAindx and SFAindx within the triglycerides. Here the model functions studied in this paper are presented. The constraints, prior knowledge used as well as several implementation details are also given.

The relations, given in Table 1, link the proton peak amplitudes ${ }^{6}$ of a lipid spectrum by introducing TG parameter variables, especially $n d b$ and $n m i d b$. These parameter variables are 1) directly fitted as they are explicitly introduced into the parameterized model function used in the first quantification approach (called $\mathrm{M}_{\text {TG_param, }}$ described below) or 2) deduced from the fitted component amplitude in the second quantification approach (called $M_{\text {peak }}$, described below), as was done in previous MRS publications ${ }^{1-4}$. Once $n d b$ and nmidb are estimated, the fatty acid composition (percentage of MUFAindx, PUFAindx and SFAindx) can be computed according to the relation given in Peterson and Mansson ${ }^{18}$ and recalled in the Appendix.

\section{$M_{\text {TG_param }}$ quantification approach}

The first $\mathrm{M}_{\mathrm{TG} \_ \text {param }}$ quantification approach studied stems from lipid composition quantification using MGE-MRI ${ }^{19}$. It is based on the following model function: 


$$
\begin{aligned}
f(t)=((\mathrm{Aw} * & n_{\text {water }} * e^{-\frac{(T E+t)}{T 2_{w}}}+A f \\
& \left.\left.* \sum_{k=1}^{8}\left(n_{k}(n d b, n m i d b) * e^{2 \pi i f_{k} t} * e^{-\frac{(T E+t)}{T 2_{k}}}\right)\right) * e^{-\frac{t}{T 2^{\prime}}}\right)
\end{aligned}
$$

127

where $A w$ is the number of water molecules, $A f$ the number of triglyceride molecules, $n_{\text {water }}$ the number of protons in a water molecule, $n_{k}(n d b, n m i d b, C L)$ the number of protons for each resonance peak, as a function of $n d b$, nmidb and $C L$ as described by Hamilton et al. ${ }^{6} . f_{k}$ is the frequency of each resonance, $T 2_{k}$ the transverse relaxation time for each peak, $T 2_{w}$ the transverse relaxation time of the water, and $T 2^{\prime}$ the global relaxation term due to $B_{0}$ heterogeneities. The water resonance was used as the reference resonance, the frequencies $f_{k}$ were set at $\left(f_{0_{k}}-4.7\right)$ * $B_{0} * \gamma / 2 \pi$ with $f_{0_{k}}$ the chemical shift (see Table 1 ) of the $\mathrm{k}^{\text {th }}$ peak (in ppm), $B_{0}$ the static magnetic field (in T) and $\gamma$ the gyromagnetic ratio of the proton (in $\mathrm{Hz} . \mathrm{T}^{-1}$ ). TE is the echo time corresponding to the localized spectroscopy sequence used. The parameter $n d b$ is the number of double bonds, nmidb the number of methylene-interrupted double bonds and $C L$ the chain length. As detailed below, these three entities, plus $A w$ and $A f$, are estimated through a fitting procedure whose implementation is the same as the quantitative MGE-MRI method given in ${ }^{19}$. Briefly in ${ }^{19}, n d b$ and $n m i d b$ are estimated separately, through three subprocesses and using strong assumptions linking $n d b, n m i d b$ and $C L$. The three subprocesses are 1) estimation of the proton density fat fraction (PDFF) and T2', which are set in the next subprocess; 2 ) estimation of $n d b$ while linking $n m i d b$ and $C L$ to $n d b$ and 3) estimation of $n m i d b$ while $n d b$ and $C L$ are linked to $n m i d b{ }^{19}$. In the last two steps, the relations between $n d b, n m i d b$ and $C L$ were: $n m i d b=0.093^{*} n d b^{2}$ and $C L=16.8+0.25^{*} n d b$ as used by Bydder et al. ${ }^{17}$. The aim of this implementation was to have a robust fitting procedure. Note that it is possible, in the implementation, not to use any connection between $n d b$ and $n m i d b$. The other parameters, especially $T 2_{k}$ and $T 2_{w}$, are set to assumed values.

When fitting in the time domain, the time samples used in the fitting procedure can be defined. Considering this point, we tested the possibility of applying the fit on undersampled data to come closer to what is performed in the quantitative MGE-MRI approaches. In these approaches, the sampling period is limited by echo spacing. Moreover, in the MGE-MRI methods, a single T2* for all peaks is fitted and there is no $\mathrm{T} 2$ weighting of the first point (expressed in equation 1 by $\exp \left(-\mathrm{TE} / \mathrm{T} 2_{\mathrm{k}}\right)$ ) because these methods are not subjected to the echo time delay of localized MR spectroscopy such as in the PRESS sequence. 
Consequently, four implementations were studied using the $M_{\text {TG_param }}$ approach [1]: 1) with full

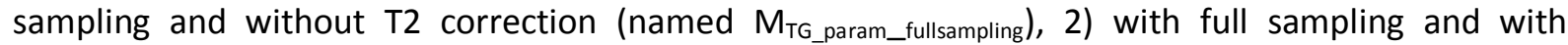

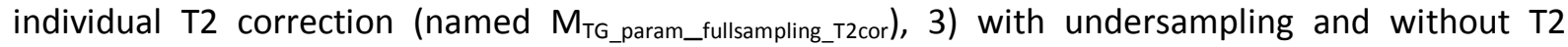

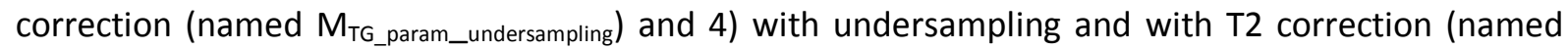
$\left.M_{T G_{-} \text {param_undersampling_T2cor }}\right)$. The undersampling of the data was defined with $t=n * t_{e}$ with $t_{e}=1 /(2 *(4.7-$ $1.3)^{*} \mathrm{~B}_{0} * \gamma / 2 \pi+$ and $\mathrm{n}=32$, while for full sampling $\mathrm{t}_{\mathrm{e}}=1 / \mathrm{BW}$ with $\mathrm{BW}$ being the spectral bandwidth used in the acquisition. We also studied the effect on TG parameter estimation of transversal decay correction. Without correction, we considered a single $T 2^{*}$ as a free parameter, i.e., all $\mathrm{T}_{\mathrm{k}}$ and $\mathrm{T} 2_{\mathrm{w}}$ were equal and the TE weighting was not taken into account, that is to say that TE was set to 0 . All these versions were integrated in homemade software written in MATLAB.

\section{$M_{\text {peak }}$ quantification approach}

The second quantification approach $\left(\mathrm{M}_{\text {peak }}\right)$ studied fits the lipid resonance peaks and the lipid composition is deduced after quantification from the relations given in Table 1. The quantification approach is based on a Voigt fitting method ${ }^{22}$, which is close to the AMARES ${ }^{10}$ method, by fitting either pure Lorentzian lines, pure Gaussian lines or a mixture of the two (Voigt lines). The model function used is described by:

$$
f(t)=e^{i \varphi 0} \sum_{k=1}^{9} c_{k} e^{\alpha_{k} \mathrm{t}+\left(\beta_{k} \mathrm{t}\right)^{2}+i 2 \pi f_{k} \mathrm{t}}
$$

where $\varphi 0$ is the zero-order phase, $c_{k}$ the amplitudes, $\alpha_{k}$ the Lorentzian damping factors, $\beta_{k}$ the Gaussian damping factors and $f_{k}$ the frequency of the $\mathrm{k}^{\text {th }}$ proton group.

The algorithm implementing $\mathrm{M}_{\text {peak }}{ }^{22}$ uses multiple random starting values for the frequency and damping factor parameters to compute, using linear least squares, the starting values of the amplitude and zero-order phase parameters as in AMARES. Then a nonlinear least squares algorithm (trust-region reflective algorithm in MATLAB) is employed to fit the global model function given in [2]. To take into account the T2 weighting on the peak amplitude, due to the localized MR spectroscopy sequence employed, the $c_{k}$ parameters were multiplied by $\exp \left(\mathrm{TE} / \mathrm{T} 2_{k}\right)$, with estimated $\mathrm{T} 2_{\mathrm{k}}$. 
181 LCModel (Version 6.3-OL, Stephen Provencher, Oakville, ON, Canada), with the control parameter 182 SPTYPE set to Lipid-8 (as described in the LCModel user's manual) was also used on experimental 183 data (described below) only. For lipid signals, this quantification method fits a flexible combination of 184 Gaussian and Lorentzian line shapes to the lipid resonances ${ }^{23}$, but the exact model function used and the implementation details are unknown. As a result, the analysis of the Monte Carlo in light of the model function and implementation used, as performed for $\mathrm{M}_{\text {peak }}$ and $\mathrm{M}_{\mathrm{TG} \_ \text {param }}$ approaches, was not allowed.

\section{Simulated data and Monte Carlo studies}

189 To compare the different quantification approaches (the four implementations with $\mathrm{M}_{\mathrm{TG} \_ \text {param }}$ and $\left.190 \mathrm{M}_{\text {peak }}\right)$, their statistical performance was assessed using simulated signals through Monte Carlo 191 studies. The two models were also compared within the Information Theory framework (known as identifiability analysis), which will be referred to as the theoretical results, with details and results given in the Appendix. The fatty acid composition of human subcutaneous abdominal adipose tissue published by Garaulet et al. ${ }^{24}$ was used as a reference in the simulated data. The targeted composition was set to $18 \%$ PUFAindx, 54.6\% MUFAindx and $27.4 \%$ SFAindx, corresponding to $n d b^{\text {target }}=2.7$ and $n m i d b^{\text {target }}=0.54$, note that in this case $n m i d b / n d b^{2}=0.074$ which was different from the relationship assumed in the fitting. The PDFF was set to $97 \%$, corresponding to Aw = 1 and Af $=37$. Four sets of Monte Carlo simulations were performed using the ten-peak signal (nine peaks for lipid and one peak for water) whose peak amplitudes are related to the fatty composition (see Table 1 and Table A1 in the Appendix). For each Monte Carlo study, a gold standard signal was designed with the target TG parameter variables and 100 Gaussian noise realizations with zero mean and a variance determined according to the desired signal-to-noise ratio (SNR) (varying from 60 to 300 in increments of 60 ) were randomly generated and added. Since several effects or imperfections, such as Voigt line shape, different T2s, or phase distortions are encountered in real acquisition, the gold standard signal was gradually complicated in the Monte Carlo studies. In the first one, the simulated signal had a simplified behavior. Indeed, all the $T 2_{\mathrm{k}}$ were assumed to be the same for all peaks resulting in a common Lorentzian damping factor $(\alpha)$ for each peak, set to $1 / \mathrm{T} 2 *$ with $\mathrm{T} 2 *$ set to $22.4 \mathrm{~ms}$ and a null Gaussian damping factor $(\beta)$. In the second set, the peak amplitudes were multiplied by $\exp \left(-\mathrm{TE} / \mathrm{T} 2_{\mathrm{k}}\right)$ with $\mathrm{TE}=14 \mathrm{~ms}$ and $\mathrm{T} 2_{\mathrm{k}}=65 \mathrm{~ms}$, and the signal was damped by a common Lorentzian damping factor $\alpha$ (which equals $1 / \mathrm{T} 2_{\mathrm{k}}$ ), and a common Gaussian damping factor $\beta$ at 
$214 \mathrm{~T} 2_{6}=29.0 \mathrm{~ms}, \mathrm{~T} 2_{7}=83.0 \mathrm{~ms}, \mathrm{~T} 2_{8}=54.7 \mathrm{~ms}, \mathrm{~T} 2_{\mathrm{w}}=300 \mathrm{~ms}$ ). For the fourth and last set, the same

215 settings as for the third set were used, with a SNR similar to the one encountered in vivo, i.e., 210.

216 Additionally, phase distortions were introduced to simulate the effect of eddy currents, as illustrated

217 in Figure 1. Finally, the absolute error mapping of $n d b$ and nmidb was computed using the same settings as used in the third set and by varying the $n d b^{\text {target }}$ and $n m i d b^{\text {target }}$ values (from 0 to 6 for $n d b$ and from 0 to 3 for $n m i d b$ ). In the following, the estimated TG parameters deduced from the $M_{\text {peak }}$ approach or fitted by the $\mathrm{M}_{\mathrm{TG} \_ \text {param }}$ approaches will be indicated with the superscript ${ }^{\text {est }}$.

\section{In vitro and in vivo data}

Eight edible oils were used for in vitro data. Their compositions were characterized by gas chromatography (details in supplementary information) performed by Functionnal Lipidomics platform of INSA and given as a pair of values ( $n d b, n m i d b)$ : avocado $(2.58,0.21)$, canola $(3.35,0.63)$, hazelnut $(2.92,0.23)$, walnut $(4.35,1.73)$, olive $(2.60,0.17)$, pistachio $(3.11,0.59)$, grape-seed $(3.89$, 1.37) and sesame $(3.17,0.78)$. In vitro MRS signals were acquired on a preclinical $4.7 T$ BioSpec Bruker system, using a PRESS sequence with $\mathrm{TR}=5000 \mathrm{~ms}, \mathrm{TE}=14.1 \mathrm{~ms}$, VOI of $4 \times 4 \times 4 \mathrm{~mm}^{3}$, one signal average and 4-kHz bandwidth, 4096 data points, without outer-volume saturation. The oil vials were $15 \mathrm{~mm}$ in diameter and $930 \mathrm{~mm}$ long. The $4 \times 4 \times 4-\mathrm{mm}^{3} \mathrm{VOI}$ was positioned in the center of the bottle. A large bandwidth of the exciting pulse was used to reduce the effect of the chemical shift artifact ( $5400 \mathrm{~Hz}$ for first pulse and $6840 \mathrm{~Hz}$ for the second and third pulses of the PRESS sequence).

Nine volunteers underwent a STEAM sequence, using respiratory triggering, on a Philips Ingenia 3T system with the following parameters: $T R=3000 \mathrm{~ms}$, multiple $T E=n * 10+4 \mathrm{~ms}$ where $n$ was an integer ranging from 1 to $6, \mathrm{TM}=16 \mathrm{~ms}, 20 \times 20 \times 20-\mathrm{mm}^{3} \mathrm{VOI}$, four signal averages, $2048-\mathrm{Hz}$ bandwidth and 1024 data points. The single voxel was located in the posterior left part of the abdominal subcutaneous adipose tissue at the level of the L4 vertebra. The MR spectrum was acquired twice in a row to perform a test and retest and to measure the repeatability of the quantification approaches. No water suppression was used. The phase of the signal of the first echo $\left(\mathrm{TE}_{1}=14 \mathrm{~ms}\right)$ was corrected with the phase of the second echo $\left(\mathrm{TE}_{2}=24 \mathrm{~ms}\right)$, which was less impacted by eddy current effects. This correction was considered possible because 1) the methylene $\left(\mathrm{CH}_{2 n}\right)$ amplitude peak was the single preponderant component and 2) $\Delta T E=T E_{2}-T_{1}$ was small compared to the lipid T2 and 1/J, where the J scalar coupling constants were between 4 and $8 \mathrm{~Hz}$ for in vivo fatty acid spectrum. The quantification methods were applied only on the spectrum of the first echo; the other echoes were acquired to estimate the T2s of each resonance peak.

For in vitro and in vivo data, no T1 relaxation correction was required, because the T1 relaxation times were much shorter than $\mathrm{TR}^{25}$. The SNR was calculated in the time domain as the ratio between 
the absolute amplitude of the real part of the first point and the standard deviation of the noise

248 calculated with the 150 last points of the signal.

249

250

251

252

253

254

255

256

257

258

259

260

261

262

263

264

265

266

267

268

269

270

271

272

273

274

275

\section{$T 2_{k}$ estimation}

For in vivo data, apparent $\mathrm{T} 2_{\mathrm{k}}$ estimation was made in the frequency domain, for each resonance, with a nonlinear least squares estimation of the monoexponential $S_{k}(T E)=S_{k}{ }^{*} \exp \left(-T E / T 2_{k}\right)$, where $S_{k}(T E)$ was the measured integrals of the $k^{\text {th }}$ peak at TE (six echoes varying regularly from $14 \mathrm{~ms}$ to 64 $\mathrm{ms}), \mathrm{SO}_{\mathrm{k}}$ the amplitude at $\mathrm{TE}=0$ and $\mathrm{T} 2_{\mathrm{k}}$ the $\mathrm{T} 2$ of the $\mathrm{k}^{\mathrm{th}}$ peak. This nonlinear regression was performed using the MATLAB Isqcurvefit function. The starting values that were used in the fitting were defined using the results of a linear fitting with the equation $\log \left(\mathrm{S}_{\mathrm{k}}(\mathrm{TE})\right)=\log \left(\mathrm{SO}_{\mathrm{k}}\right) *\left(-\mathrm{TE} / \mathrm{T} 2_{\mathrm{k}}\right)$. For in vitro data, the $\mathrm{T} 2_{\mathrm{k}}$ were set at $\mathrm{T} 2_{1}=40.8 \mathrm{~ms}, \mathrm{~T} 2_{2}=17.5 \mathrm{~ms}, \mathrm{~T} 2_{3}=48.6 \mathrm{~ms}, \mathrm{~T} 2_{4}=27.7 \mathrm{~ms}, \mathrm{~T} 2_{5}=34.0 \mathrm{~ms}$, $\mathrm{T} 2_{6}=17.6 \mathrm{~ms}, \mathrm{~T} 2_{7}=49 \mathrm{~ms}, \mathrm{~T} 2_{8}=37.9 \mathrm{~ms}^{26}$.

\section{Comparison of the quantification results}

For the Monte Carlo studies, the quantification results were compared to the theoretical parameter values used to generate the signal and biases, and the variabilities on the TG parameter estimations were assessed and compared. For the oil acquisitions, the quantification results were compared according to their concordance to the known oil composition. For the SAT in vivo acquisitions, for each index or parameter of interest and each subject, a percent difference was calculated as the ratio between the absolute difference between the two estimations and the mean of these two estimations. The variability percentage $(\operatorname{Var})$ of the test-retest was then the average of these percent differences computed on the nine subjects $(n=9)$ :

$\operatorname{Var}=\frac{1}{\mathrm{n}} \sum_{i=1}^{N} \frac{\mid \text { test }_{i}-\text { retest }_{i} \mid}{\left(\text { test }_{i}+\text { retest }_{i}\right) / 2} * 100 \%$

where test $_{i}$ and retest $_{i}$ are the estimation and second estimation, respectively, of one of the parameters of interest ( $n d b$, nmidb, MUFAindx, PUFAindx, SFAindx). For each index or parameter of interest, the average of the two estimations was then compared to the fatty acid composition found in the literature ${ }^{24,27,28}$.

In vivo spectra before and after eddy current correction and in vitro spectra were also quantified with LCModel, for comparison with $M_{\text {TG_param }}$ quantification versions and $M_{\text {peak }}$ quantification on the experimental data. 


\section{Visual fitting results vs quantitative results}

Figure 2 shows the fitting results of two different methods (LCModel and $M_{\text {peak }}$ ) applied on the same in vivo spectrum. In both cases, the residual signal (i.e., the difference between the fitted spectrum and the original spectrum) appears to be very small and similar, but the quantitative results differ noticeably between the two methods (3.8\% PUFAindx, 43\% MUFAindx and 53.6\% SFAindx with the Voigt model and 9.6\% PUFAindx, 50\% MUFAindx and 40.5\% SFAindx with LCModel), hence the need to quantify the methods to be studied in terms of their statistical performance (bias and variance on the parameter estimation) and their sources of instability or error.

\section{Results of the Monte Carlo studies}

The results of the first Monte Carlo study, which mimicked in vivo acquisitions, were consistent with the identifiability analysis given in the Appendix. The standard deviation (SD) of the relative difference between the estimated value and the target value (which is proportional to the percentage root mean square error) was compared with the theoretical uncertainty (based on the Cramér Rao Lower Bounds computation) derived from quantification approaches including subprocessing. Regarding $n d b$, the SD was $1.04 \%$ for the $\mathrm{M}_{\text {TG_param_fullsampling, } 1.46 \% \text { for }}$

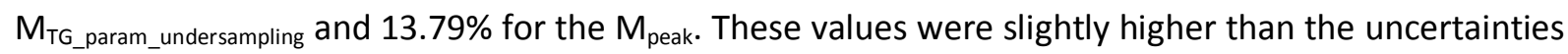
computed in the identifiability analysis (Table A2 in the Appendix) - 0.9\% for the $\mathrm{M}_{\text {TG_param_fullsampling, }}$ 1.35\% for the $\mathrm{M}_{\text {TG_param_undersampling }}$ and $4.89 \%$ for the $\mathrm{M}_{\text {peak }}$ - but they showed the same trend. The

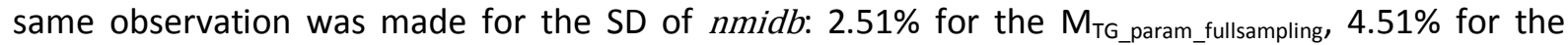

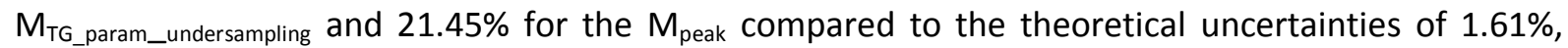
$2.45 \%$ and $18.98 \%$, respectively. We observed the same tendency for the other Monte Carlo simulations.

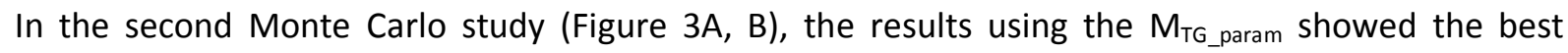
results with the smallest variability but had a bias of $-6.34 \pm 0.11 \%$ for $n d b$ (respectively, $42.05 \pm$ $0.35 \%$ for $n m i d b$ ) for the undersampling and a bias of $-20.28 \pm 0.08 \%$ (respectively, $-20.18 \pm 0.17 \%$ for $n$ midb) for the full sampling. The $\mathrm{M}_{\text {peak }}$ quantification provided the lowest biased estimations (bias of $0.72 \pm 0.78 \%$ for $n d b$ and $-0.78 \pm 4.77 \%$ for $n m i d b)$ but with higher variability. Here, as one can expect, when all peaks have the same T2 relaxation times, the introduction of T2 as a priori knowledge in the $\mathrm{M}_{\mathrm{TG} \_ \text {param }}$ quantification has no effect on the estimation. On the contrary, when the simulated data have different T2 values for each peak as in the third Monte Carlo study (Figure 3C, D), the introduction of T2 a priori knowledge in the $\mathrm{M}_{\mathrm{TG} \text { _param }}$ approach reduces the absolute bias for both undersampling and full sampling. In the last Monte Carlo study (Figure 3E, F), the fitting using 
the $M_{\text {peak }}$ approach was greatly influenced by the distortion of the baseline, unlike the $M_{\text {TG_param }}$. The estimation of $n m i d b$ was the most influenced, with a bias of up to $400 \%$. When all lipid peaks had different T2s, the M $\mathrm{M}_{\mathrm{TG} \_ \text {param }}$ quantification with undersampling and T2 correction had the smallest variability but some biased estimated values. This bias could also be influenced by the assumed relation between $n d b$ and $n m i d b$, here $n m i d b=0.093^{*} n d b^{2}$ while the ones used in the simulation are different. Besides the pair of values - ndb and nmidb - which moved off this relation showed larger biases, especially for the estimation of nmidb (see Figure 4).

\section{In vitro results}

The mean SNR for in vitro data was 2062 (range, 1769-2360). In the particular case of in vitro data, where the MR spectroscopic signal show high SNR and good spectral resolution in the implementation of $\mathrm{M}_{\text {TG_param }}$, the constraint linkink $n d b$ and nmidb has not been used as the relationship ( $n m i d b=0.093^{*} n d b^{2}$ ) was not found for all oils in the results from gas chromatography. In the present case, nmidb was freely fitted in the last step, which enabled to improve the accuracy of $\mathrm{M}_{\mathrm{TG} \_ \text {param }}$ approach results. Figure 5 shows the oil quantification results taking the composition characterized by gas chromatography as the gold standard reference. For each quantification approach studied, estimated values correlated well with the theoretical values (with a coefficient of determination $r^{2}$ close to 1$)$. However, $M_{\text {peak }}$ quantification slightly underestimated $n d b$ and $\mathrm{M}_{\mathrm{TG} \_ \text {param_undersampling }}$ overestimated it. The $\mathrm{M}_{\mathrm{TG} \_ \text {param_undersampling_T2cor }}$ (with a priori knowledge on T2s included in the model) gave good estimation of $n d b$, as well as LCModel. For nmidb, Mpeak gave the best estimation, $\mathrm{M}_{\mathrm{TG} \_ \text {param_undersampling_T2cor }}$ and LCModel slightly overestimated nmidb. In the range of expected in vivo nmidb (0.30-1.0), LCModel and M M_param_undersampling seemed to give a good estimation of the nmidb value.

\section{In vivo results}

The nine male volunteers, aged $26.1 \pm 6.3$ years, had a BMI of $24.8 \pm 1.4 \mathrm{~kg} / \mathrm{m}^{2}$. The SNR of the MR spectra varied between 203 and 314. Of the 18 acquisitions (test and retest acquisitions together) the mean and the standard deviation of $\mathrm{T} 2_{\mathrm{k}}$ were the following: $\mathrm{T} 2_{1}=47.3 \pm 3.0 \mathrm{~ms}, \mathrm{~T} 2_{2}=30.5 \pm 2.3$ $\mathrm{ms}, \mathrm{T} 2_{2 \mathrm{~b}}=34.3 \pm 2.4 \mathrm{~ms}, \mathrm{~T} 2_{3}=45.0 \pm 17.1 \mathrm{~ms}, \mathrm{~T} 2_{4}=41.8 \pm 4.6 \mathrm{~ms}, \mathrm{~T} 2_{5}=35.8 \pm 1.7 \mathrm{~ms}, \mathrm{~T} 2_{7}=83.0 \pm$ $2.4 \mathrm{~ms}, \mathrm{~T} 2_{8}=54.7 \pm 6.1 \mathrm{~ms}$. T2 ${ }_{6}$ was not estimated because the peak at $1.6 \mathrm{ppm}$ was indistinguishable from the peak at $1.3 \mathrm{ppm}$. $\mathrm{T} 2_{\mathrm{k}}$ values were used in the $\mathrm{M}_{\mathrm{TG} \_ \text {param }}$ quantification to correct estimated amplitudes of the $M_{\text {peak }}$ and LCModel quantification.

For the in vivo results (Table 2), the test-retest variability was the smallest for the

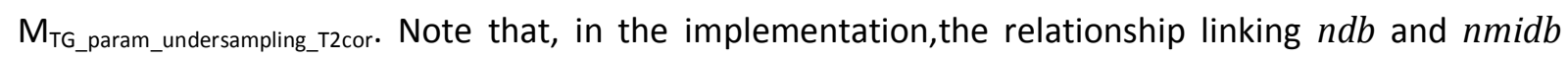
enabled the $\mathrm{M}_{\text {TG_param_undersampling_T2cor }}$ fitting approach to reduce the test-retest variability on in vivo 
spectra ( $22.3 \%$ vs $4.1 \%$ for $n$ midb estimation and $7.3 \%$ vs $2.0 \%$ for $n d b$ estimation). This model (using or not the ndb-nmidb connection) seemed to give values close to the literature values taken as reference. LCModel showed good test-retest variability- similar to the one obtained with MTG_param_undersampling_T2cor and a ndb-nmidb connection in its implementation but with different estimated parameter values.

LCModel was applied on the spectrum at the first TE and the results were compared with and without phase correction. The mean estimated values of $n d b$, nmidb, PUFAindx, MUFAindx and SFAindx were equivalent in both cases: the values without correction were equal to $1.49 \pm 0.18,0.39$ $\pm 0.08,13.0 \pm 2.6 \%, 23.6 \pm 9.5 \%, 63.4 \pm 7.5 \%$, respectively, compared to the values in Table 2 . However, the test-retest variability percentage was better with the phase correction than without this correction: $2.0 \%$ vs $4.5 \%$ for $n d b, 7.6 \%$ vs $15.2 \%$ for $n m i d b, 7.6 \%$ vs $15.2 \%$ for PUFAindx, $4.7 \%$ vs $12.5 \%$ for MUFAindx and $4.0 \%$ vs $8.3 \%$ for SFAindx.

\section{Discussion}

Lipid MRS is a simple and fast tool to analyze metabolic modification when quantifying fatty acid: it could be advantageous in the follow-up of the lipid composition modification in adipose tissues in weight gain or weight loss phases, in the evaluation of fat surrounding a tumor or in inflammatory tissue ${ }^{23,29}$. However, the fatty composition modifications could be very small in comparison to the measurement uncertainties related to the current methods. Moreover, as often encountered in quantitative MRI or MRS, validation of the quantification method taking into account the reproducibility on different imaging systems from different manufacturers is a major problem. This paper has demonstrated that, in the particular case of in vivo lipid signal analysis, the M $_{\text {TG_param }}$ approach could be a solution to be considered for fatty acid composition assessment as thisfitting solution appears to be robust to in vivo conditions .

The $M_{\text {TG_param }}$ quantification approach, where there is a linear relationship between the estimated parameters and the proportion of fatty acid types to be determined, has been qualified as a direct estimation method. With this approach, the relative error in the estimation of fatty acid composition would be the same as that of the estimated parameter (e.g., nmidb with PUFAindx) or the sum of parameter errors (e.g., MUFAindx and SFAindx). In the $M_{\text {peak }}$ approach, the fatty acid composition is determined by ratios of peak amplitudes ${ }^{1-4}$, which is qualified as an indirect determination. In this case, small errors on the estimation of two peak amplitudes would increase errors and therefore result in greater variability of the results. 
The variability of the results could be anticipated by experimental design, by studying the properties of the model function employed (calculating the condition number of the Jacobian matrix) as well as the theoretical uncertainties, as described in the Appendix. A too large condition number expresses an ill-conditioned problem and the parameters to estimate cannot be solely determined from the signal acquired, as for the $M_{\text {peak }}$ approach, which also showed higher theoretical uncertainties than the $M_{\text {TG_param }}$ approach. The same observations were made in the Monte Carlo studies for the variability of the different approaches. The test-retest variabilities obtained with the in vivo data also corroborate these assessments and allow saying that $\mathrm{M}_{\mathrm{TG} \text { _param }}$ provides robust with small test-retest variability percentage estimates. This small variability percentage is mainly due to the relationship linking $\mathrm{ndb}$ and nmidb with a quadratic function which narrows the scope of the solutions while letting some latitude in the fit results. If no constraints or relationship are imposed, ndb and nmidb are correlated and higher variability is found. .

As a result, in the particular case of adipose tissue, in vivo MRS lipid quantification, the model function simplification used in the $\mathrm{M}_{\mathrm{TG} \_ \text {param }}$ appears to be a key leverage point to increase the precision of the result, at the expense of a possible bias.

\section{Bias considerations}

Reducing the number of parameters enabled us to decrease the variability of the results but this might have induced a bias in estimating the fatty acid composition. We investigated several sources of error and several implementations of $\mathrm{M}_{\mathrm{TG} \_ \text {param }}$ that could bias the estimations. With the Monte Carlo studies, biases on $n d b$ and especially nmidb estimations were observed because the relationship between $n d b$ and $n m i d b$ assumed in the fitting algorithm was not checked in the parameters used to simulate the signal. This mismatch was implemented on purpose to underline the importance of the assumption linking $n d b$ and nmidb in the $\mathrm{M}_{\mathrm{TG} \_ \text {param }}$ approach, which participates in the robustness of the method but also constitutes a limitation of the method. Biased estimations could also occur if lipid multi-peak T2 weightings are simplified to a single lipid T2 in the fitting model. When different T2 weightings were used for the different lipid peaks involved in the TG parameter variables (as was case on in vivo data), their inclusion in the computation process appeared to be important to avoid bias in estimating the fatty acid composition. For LCModel and $M_{\text {peak, }}$, the amplitudes need correcting by the factor $\exp \left(T E / T 2_{k}\right)$ prior to estimating the fatty acid composition, as emphasized earlier ${ }^{2,3}$. Of course, the correction is unnecessary if the two resonance peaks used in the $n d b$ or $n m i d b$ relation have the same $T 2$ relaxation time. For $\mathrm{M}_{\mathrm{TG} \_ \text {param, }}$ the $T 2_{\mathrm{K}}$ correction needs to be included in the model and $\mathrm{T} 2{ }_{\mathrm{k}} \mathrm{s}$ need to be estimated before. Note that the contribution of glycerol (5.19 ppm) was considered to be part of the olefinic contribution (5.29 ppm) 
because these two peaks were too close to be distinguished. Therefore, we considered that the two peaks had the same $\mathrm{T} 2$ relaxation time. Considering that $\mathrm{T} 2$ weighting can bias the estimations of the fatty acid composition, it could be advantageous to use an ultra-short echo time sequence ${ }^{30}$. It should also be noted that the PUFAindx calculation, here made from nmidb, was not an absolute quantification but a coherent index of polyunsaturation, in contrast to the other types of fatty acid. Using this calculation, the maximum possible proportion of PUFA is calculated. When the most frequently identified PUFA was di-unsaturated fatty acid, this PUFAindx calculation led to consistent results. On the other hand, it could be possible to quantify the proportion of PUFA more precisely by calculating the proportion of $\omega-3^{31,32}$ and correcting the present calculation.

In this case, the effect of T2 weighting will have to be considered. In this study, we focused on the quantification of fatty acids where lipids were largely predominant (PDFF 95\% in oil and in adipose tissue). In fat/water mixtures, Perterson et al. ${ }^{33}$ showed that the most critical point to achieve a reduction of errors for the quantitative imaging method was to correctly estimate the T2 of water and the 1.3-ppm peak. This would also be true for the $\mathrm{M}_{\mathrm{TG} \_ \text {param }}$ approach.

In the case of fat liver quantification, Hamilton et al. ${ }^{34}$ showed that the fatty acid quantification results depend on the ${ }^{1} \mathrm{H}$ MRS sequence used to collect data. Fat peak areas normalized by the water peak were consistently greater on PRESS than on STEAM and the relative amplitudes of the methyl and methylene peaks were found different in STEAM compared to PRESS due to different apparent T2 correction. Note that these considerations also depend on the quantification method used (in this case AMARES). Consequently, Hamilton et al. ${ }^{7}$ used the STEAM sequence, with mixing time and echo time optimized to minimize J-coupling effect, to estimate the adipose tissue fatty acid composition. In the present in vitro study, estimations of $n d b$ and nmidb, corrected with $\mathrm{T} 2$ values, were close to the theoretical values for all the fitting approaches, which suggests that fatty acid composition can also be estimated using the PRESS sequence. It has also been demonstrated that PRESS or STEAM can yield consistent fatty acid composition by using long echo time ( $\sim 120 \mathrm{~ms}$ for STEAM and $\sim 180 \mathrm{~ms}$ for PRESS) ${ }^{32}$. In this latter case, the optimized echo time for each sequence has been experimentally defined at $3 T$ to match high resolution NMR measurements. The undersampling used in the $\mathrm{M}_{\text {TG_param_undersampling }}$ method focused the analysis on the methylene peak time domain evolution. For this method, the importance of taking into account the spectral pattern due to J-coupling modulation is less critical.

The time domain quantification approach can go back and forth between the full sampling (enabling the spectrum to be inspected after Fourier transform) and the constrained sampling achieved in MGE-MRI. On simulated data with different T2s for each peak, it was demonstrated that the $\mathrm{M}_{\text {TG_param }}$ 
using a regular undersampling reduced bias. Undersampling was also found to reduce bias 1 ) when the parameterized model function had pure Lorentzian damping factors while the data to adjust had a Voigt line shape (i.e., a mixture of Gaussian and Lorentzian damping factors) and 2) when the data presented phase distortions. Note that undersampling was made possible in $\mathrm{M}_{\mathrm{TG} \_ \text {param }}$ because the number of parameters to estimate was reduced. The undersampling method used in this paper concentrated the least squares minimization on data samples with a good SNR, as the frequency sampling was set on the methylene peak frequency. Moreover, since it resulted in fewer data samples in the minimization procedure, the estimates are less influenced by the mismatch of the exponentially decaying envelop between the model and the original data.

Undersampling also reduces the processing time. For example, in this study, on the in vivo spectrum the $\mathrm{M}_{\mathrm{TG} \_ \text {param_undersampling }}$ had a processing time of around $10 \mathrm{~s}$ versus $25 \mathrm{~s}$ for $\mathrm{M}_{\mathrm{TG} \_ \text {param_fullsampling }}$ and versus $80 \mathrm{~s}$ for our implementation of $\mathrm{M}_{\text {peak }}$. The time domain fitting approach could also be useful in future investigations for irregular sampling of the acquisition ${ }^{35,36}$.

Validating a quantification strategy is a difficult task. The methodology proposed to study and validate different quantification strategies has limitations inherent to the use of simulated/in vitro/in vivo data. Monte Carlo simulation analysis is always biased because the model and variations studied are necessarily only an approximation and simplification of real-life data. Nevertheless, Monte Carlo simulation analysis has the advantage of studying possible sources of error separating those leading to variability and increased uncertainties from those resulting in systematic under- or overestimation (bias). The in vitro validation also shows limitations for the validation of a quantification strategy that aimed to accurately fit in vivo acquired data. Indeed, in vitro acquisitions show a different spectral pattern from in vivo acquisitions due to different homogeneity field conditions. As a result, the line shape is different from in vivo spectra and the resonating groups depict multiplets that are better spectrally resolved. Nevertheless, this work shows that the standard MRS method ( $M_{\text {peak }}$ and LCmodel) lead to valuable results on in vitro oil acquisitions. Validation of the in vivo results is also a difficult task: a strict gold standard could be a countermeasure provided, for example, by gas chromatography analysis ${ }^{26}$. This technique should be performed with selective analysis of major classes of lipids (triglyceride, phospholipid, cholesterol) so that it can be easily compared to MRS measurements. However, this technique requires biopsies, which can be unjustified from an ethical point of view when working, as here, on human volunteers. Gas chromatography analysis would be feasible in the context of future animal studies. To validate these results, we opted for a repeatability 
assessment (with a test-retest approach) and for a likelihood assessment by comparing the

472 estimated lipid composition with Garaulet et al. ${ }^{24}$, Hodson et al. ${ }^{27}$ and Field et al. ${ }^{28}$.

473 Quantification results obtained with LCModel software, widely used in the in vivo MRS community 47423,37 , were included. The results obtained by LCModel seem to present good in vitro and in vivo 475 properties, with good repeatability, providing a seemingly plausible fatty acid composition. By 476 looking at the LCModel results and fits, it is most probable that the LCModel quantification strategy 477 employed regularization to handle small baseline variations (due to a possible eddy current effect at 478 short echo times or short first-order phases). In our view, this regularization should have been done 479 in a specific way, i.e., based on prior knowledge of the relative peak amplitudes, but the exact 480 implementation of this is unknown.

481 It should be noted that what matters most in the quantification process is the correct (i.e., with the 482 least variability and least bias) estimation of peak amplitude parameters. However, a strong 483 interdependence exists in the estimation of the peak amplitude parameter and the estimation of the corresponding lineshape/damping parameters. The key question is how to handle this interdependence while mathematically converging to a solution that has a physical meaning. Moreover, the estimation should be repeatable, robust to some signal variation; would it be noise or small phase/baseline variation. The final estimates are expected to be sufficiently sensitive to detect possible disease-related metabolic variations. When adopting a model that is intended to fit the whole lipid spectrum pattern, the parameters are so dependent on each other that the inverse problem to solve becomes increasingly ill-conditioned. Two alternatives are then possible: a) either simplify the model, in other words reduce the number of parameters to fit with sufficient and correct prior knowledge to alleviate multicollinearity effects resulting from correlated parameters or b) find a regularization strategy that can handle the ill-posed problem (as most probably performed by LCModel). The ndb-nmidb quadratic link ${ }^{17}$, assumed in $\mathrm{M}_{\text {TG_param }}$ might hide physiologically relevant information. As the result, the user is advised to carefully question the constraints and prior knowledge used in the fitting procedure.

\section{Conclusion}

498 A Quantification approach inspired from quantitative MGE-MRI has been compared to standard MRS 499 method and shows interesting properties in terms of robustness and test-retest variability, at the 500 expense of a possible bias. This work contributes to assembling the quantification approach used in quantitative MRI and the historical gold standard spectroscopy. 
Acknowledgments: This study was conducted as part of the LABEX PRIMES (ANR-11-LABX-

504

505

506

507

508

509

510

511

512 0063) of the "Université de Lyon", within the "Investissements d'Avenir" program (ANR-11IDEX-0007) operated by the French National Research Agency (ANR). In vitro acquisitions were performed on the PILoT platform, member of the France Life Imaging network (grant ANR-11-INBS-0006). This study was also supported by the IHU OpéRa (ANR-10-IBHU-0004), within the "Investissements d'Avenir"program operated by the French National Research Agency (ANR) and PHRC-IR Visfatir. 


\section{Theoretical comparison of the quantification approaches}

Here, an approach based on Information Theory is used for theoretical comparison of the $\mathrm{M}_{\text {TG_param }}$ and $\mathrm{M}_{\text {peak }}$ approaches. For each model function, realistic parameter values were used (described in Table A2) to correspond to in vitro and in vivo conditions. The condition number of the Jacobian matrix (cond-J), the correlation matrix and the parameter uncertainty $\frac{\Delta \theta}{\theta}$ were computed (the relative CRLBs $\frac{\Delta \theta}{\theta}=\sigma_{0} * \frac{\sqrt{F(\theta)^{-1}}}{\theta}$, where $\theta=\left\{\right.$ ndb, nmidb or $\left.c_{1}, c_{3}, c_{8}\right\}, \quad F^{-1}=\mathfrak{R}\left(J^{T} . J\right)^{-1}$; the inverse Fisher matrix and $\sigma_{0}$ the standard deviation of noise, and for PUFAindx, MUFAindx and SFAindx the calculation is detailed in Table A1).

The resonance frequencies $f_{k}$, relaxation times $T 2_{w}$ and $T 2_{k}$ were assumed to be fixed and equal as if they had been previously estimated. TE was set to 0 . For the $M_{T G_{-} \text {param }}$ quantification case, five parameters (Aw, Af, $n d b, n m i d b, \mathrm{~T}^{*}$ ) were considered as estimated. For the $\mathrm{M}_{\text {peak }}$ quantification, $2 * 9$ parameters $\left(\alpha_{1}, \ldots, \alpha_{9}, c_{1}, \ldots, c_{9}\right)$ were considered as estimated. To be sure that the two models described mathematically the same signal and to simplify the comparison, for the $M_{\text {peak }}$ quantification we used the following parameters: $\varphi 0=0, \alpha_{k}=-\left(\frac{1}{T 2^{\prime}}+\frac{1}{T 2_{k}}\right)=-\frac{1}{T 2^{*}}, \beta_{k}=$ $0, c_{k}=A_{f} * n_{k}(n d b, n m i d b, C L)$ for $k=\{1, \ldots, 8\}, c_{9}=A_{w} * n_{w a t e r}, \alpha_{9}=-\left(\frac{1}{T 2^{\prime}}+\frac{1}{T 2_{w}}\right)=$ $-\frac{1}{T 2^{*}}$.

The results on this theoretical model comparison are summarized in Table A2. The condition number of the $M_{\text {peak }}$ were $10^{\wedge} 3$ times higher than the condition number of the $M_{T G \_p a r a m}$. As expected, the uncertainties increased when the condition number was higher, so the $M_{\text {peak }}$ had uncertainties higher than the $\mathrm{M}_{\mathrm{TG} \_ \text {param. }}$. The segmented process of the $\mathrm{M}_{\mathrm{TG} \_ \text {param }}$ gave uncertainties lower than if we estimated all the parameters all at once (Table A2). The condition number of the M TG_param $_{\text {was }}$ greatly influenced by the Af and Aw values without impacting the uncertainty values of $n d b$ and $n$ midb. It appeared that if we normalized the Af and Aw values by the factor $(1 /(A f+A w))$, the condition number was lower, so we used this correction for the results summarized in Table A2. The correlation matrix of the $\mathrm{M}_{\text {peak }}$ indicated that $\alpha_{k}$ and $c_{k}$ were strongly correlated with a Pearson coefficient ( $r$ ) of 0.71 for each resonance. For the $\mathrm{M}_{\mathrm{TG} \_ \text {param }}$ with no constraint on parameters $n d b$ and $n m i d b$, the correlation matrix showed a strong correlation between $n d b$ and $n m i d b(r=0.80)$, and a moderate correlation between Af and $n d b(r=0.62)$, Af and nmidb $(r=0.42)$, and Af and $T 2^{\prime}(r=0.41)$. 


\section{References}

1. Zancanaro C, Nano R, Marchioro C, Sbarbati A, Boicelli A, Osculati F. Magnetic resonance spectroscopy investigations of brown adipose tissue and isolated brown adipocytes. J Lipid Res. 1994;35(12):2191-2199.

2. Ren J, Dimitrov I, Sherry AD, Malloy CR. Composition of adipose tissue and marrow fat in humans by $1 \mathrm{H}$ NMR at 7 Tesla. J Lipid Res. 2008;49(9):2055-2062. doi:10.1194/jlr.D800010JLR200.

3. Strobel K, Hoff J van den, Pietzsch J. Localized proton magnetic resonance spectroscopy of lipids in adipose tissue at high spatial resolution in mice in vivo. $J$ Lipid Res. 2008;49(2):473-480. doi:10.1194/jlr.D700024-JLR200.

4. Corbin IR, Furth EE, Pickup S, Siegelman ES, Delikatny EJ. In vivo assessment of hepatic triglycerides in murine non-alcoholic fatty liver disease using magnetic resonance spectroscopy. Biochim Biophys Acta BBA - Mol Cell Biol Lipids. 2009;1791(8):757-763. doi:10.1016/j.bbalip.2009.02.014.

5. Lundbom J, Hakkarainen A, Fielding B, Söderlund S, Westerbacka J, Taskinen M-R, Lundbom N. Characterizing human adipose tissue lipids by long echo time $1 \mathrm{H}-\mathrm{MRS}$ in vivo at 1.5 Tesla: validation by gas chromatography. NMR Biomed. 2010;23(5):466-472. doi:10.1002/nbm.1483.

6. Hamilton G, Yokoo T, Bydder M, Cruite I, Schroeder ME, Sirlin CB, Middleton MS. In vivo characterization of the liver fat $1 \mathrm{H}$ MR spectrum. NMR Biomed. 2011;24(7):784-790. doi:10.1002/nbm.1622.

7. Hamilton G, Schlein AN, Middleton MS, Hooker CA, Wolfson T, Gamst AC, Loomba R, Sirlin CB. In vivo triglyceride composition of abdominal adipose tissue measured by $1 \mathrm{H}$ MRS at $3 \mathrm{~T}$. $J$ Magn Reson Imaging. 2017;45(5):1455-1463. doi:10.1002/jmri.25453.

8. Machann J, Stefan N, Wagner R, Bongers M, Schleicher E, Fritsche A, Häring H-U, Nikolaou K, Schick F. Intra- and interindividual variability of fatty acid unsaturation in six different human adipose tissue compartments assessed by 1H-MRS in vivo at 3 T. NMR Biomed. 2017;30:e3744. doi:10.1002/nbm.3744.

9. Lundbom J, Hakkarainen A, Söderlund S, Westerbacka J, Lundbom N, Taskinen M-R. Long-TE 1H MRS suggests that liver fat is more saturated than subcutaneous and visceral fat. NMR Biomed. 2011;24(3):238-245. doi:10.1002/nbm.1580.

10. Vanhamme L, van den Boogaart A, Van Huffel S. Improved Method for Accurate and Efficient Quantification of MRS Data with Use of Prior Knowledge. J Magn Reson. 1997;129(1):35-43. doi:10.1006/jmre.1997.1244.

11. Provencher SW. Estimation of metabolite concentrations from localized in vivo proton NMR spectra. Magn Reson Med. 1993;30(6):672-679. doi:10.1002/mrm.1910300604.

12. Provencher SW. Automatic quantitation of localized in vivo1H spectra with LCModel. NMR Biomed. 2001;14(4):260-264. doi:10.1002/nbm.698. 
13. Poullet J-B, Sima DM, Simonetti AW, De Neuter B, Vanhamme L, Lemmerling P, Van Huffel S. An automated quantitation of short echo time MRS spectra in an open source software environment: AQSES. NMR Biomed. 2007;20(5):493-504. doi:10.1002/nbm.1112.

14. Osorio-Garcia MI, Sima DM, Nielsen FU, Himmelreich U, Van Huffel S. Quantification of magnetic resonance spectroscopy signals with lineshape estimation. J Chemom. 2011;25(4):183-192. doi:10.1002/cem.1353.

15. Ratiney H, Coenradie Y, Cavassila S, Ormondt D van, Graveron-Demilly D. Time-domain quantitation of $1 \mathrm{H}$ short echo-time signals: background accommodation. Magn Reson Mater Phys Biol Med. 2004;16(6):284-296. doi:10.1007/s10334-004-0037-9.

16. Mosconi E, Sima DM, Osorio Garcia MI, Fontanella M, Fiorini S, Van Huffel S, Marzola P. Different quantification algorithms may lead to different results: a comparison using proton MRS lipid signals. NMR Biomed. 2014;27(4):431-443. doi:10.1002/nbm.3079.

17. Bydder M, Girard O, Hamilton G. Mapping the double bonds in triglycerides. Magn Reson Imaging. 2011;29(8):1041-1046. doi:10.1016/j.mri.2011.07.004.

18. Peterson $P$, Månsson S. Simultaneous quantification of fat content and fatty acid composition using MR imaging. Magn Reson Med. 2013;69(3):688-697. doi:10.1002/mrm.24297.

19. Leporq B, Lambert SA, Ronot M, Vilgrain V, Van Beers BE. Quantification of the triglyceride fatty acid composition with 3.0T MRI. NMR Biomed. 2014;27(10):1211-1221. doi:10.1002/nbm.3175.

20. Leporq B, Lambert SA, Ronot M, Boucenna I, Colinart P, Cauchy F, Vilgrain V, Paradis V, Van Beers BE. Hepatic fat fraction and visceral adipose tissue fatty acid composition in mice: Quantification with 7.0T MRI. Magn Reson Med. 2016;76(2):510-518. doi:10.1002/mrm.25895.

21. Leporq B, Lambert S a., Ronot M, Vilgrain V, Van Beers B e. Simultaneous MR quantification of hepatic fat content, fatty acid composition, transverse relaxation time and magnetic susceptibility for the diagnosis of non-alcoholic steatohepatitis. NMR Biomed.:n/a-n/a. doi:10.1002/nbm.3766.

22. Ratiney H, Bucur A, Sdika M, Beuf O, Pilleul F, Cavassila S. Effective voigt model estimation using multiple random starting values and parameter bounds settings for in vivo hepatic $1 \mathrm{H}$ magnetic resonance spectroscopic data. In: 2008 5th IEEE International Symposium on Biomedical Imaging: From Nano to Macro. ; 2008:1529-1532. doi:10.1109/ISBI.2008.4541300.

23. Coum A, Ouldamer L, Noury F, Barantin L, Saint-Hilaire A, Vilde A, Bougnoux P, Gambarota G. In vivo MR spectroscopy of human breast tissue: quantification of fatty acid composition at a clinical field strength (3 T). Magn Reson Mater Phys Biol Med. 2016;29(1):1-4. doi:10.1007/s10334-015-0506-3.

24. Garaulet M, Hernandez-Morante JJ, Lujan J, Tebar FJ, Zamora S. Relationship between fat cell size and number and fatty acid composition in adipose tissue from different fat depots in overweight/obese humans. Int J Obes. 2006;30(6):899-905. doi:10.1038/sj.ijo.0803219.

25. Brix G, Heiland S, Bellemann ME, Koch T, Lorenz WJ. MR imaging of fat-containing tissues: Valuation of two quantitative imaging techniques in comparison with localized proton spectroscopy. Magn Reson Imaging. 1993;11(7):977-991. doi:10.1016/0730-725X(93)90217-2. 
26. Ramamonjisoa N, Ratiney H, Mutel E, Guillou H, Mithieux G, Pilleul F, Rajas F, Beuf O, Cavassila S. In vivo hepatic lipid quantification using MRS at 7 Tesla in a mouse model of glycogen storage disease type 1a. J Lipid Res. 2013;54(7):2010-2022. doi:10.1194/jlr.D033399.

27. Hodson L, Skeaff CM, Fielding BA. Fatty acid composition of adipose tissue and blood in humans and its use as a biomarker of dietary intake. Prog Lipid Res. 2008;47(5):348-380. doi:10.1016/j.plipres.2008.03.003.

28. Field CJ, Angel A, Clandinin MT. Relationship of diet to the fatty acid composition of human adipose tissue structural and stored lipids. Am J Clin Nutr. 1985;42(6):1206-1220.

29. Dorez H, Ratiney H, Canaple L, Saint-Jalmes H, Gaillard S, Moussata D, Sablong R, Beuf O. In vivo MRS for the assessment of mouse colon using a dedicated endorectal coil: initial findings. NMR Biomed. 2017;30:e3794. doi:10.1002/nbm.3794.

30. Gajdošík M, Chadzynski GL, Hangel G, Mlynárik V, Chmelík M, Valkovič L, Bogner W, Pohmann $\mathrm{R}$, Scheffler K, Trattnig S, Krššák M. Ultrashort-TE stimulated echo acquisition mode (STEAM) improves the quantification of lipids and fatty acid chain unsaturation in the human liver at $7 \mathrm{~T}$. NMR Biomed. 2015;28(10):1283-1293. doi:10.1002/nbm.3382.

31. Lundbom J, Heikkinen S, Fielding B, Hakkarainen A, Taskinen M-R, Lundbom N. PRESS echo time behavior of triglyceride resonances at 1.5T: Detecting $\omega-3$ fatty acids in adipose tissue in vivo. $J$ Magn Reson. 2009;201(1):39-47. doi:10.1016/j.jmr.2009.07.026.

32. Fallone CJ, McKay RT, Yahya A. Long TE STEAM and PRESS for estimating fat olefinic/methyl ratios and relative $\omega-3$ fat content at 3T. J Magn Reson Imaging.:n/a-n/a. doi:10.1002/jmri.25920.

33. Peterson $\mathrm{P}$, Svensson J, Månsson S. Relaxation effects in MRI-based quantification of fat content and fatty acid composition. Magn Reson Med. 2014;72(5):1320-1329. doi:10.1002/mrm.25048.

34. Hamilton G, Middleton MS, Bydder M, Yokoo T, Schwimmer JB, Kono Y, Patton HM, Lavine JE, Sirlin CB. Effect of PRESS and STEAM sequences on magnetic resonance spectroscopic liver fat quantification. J Magn Reson Imaging. 2009;30(1):145-152. doi:10.1002/jmri.21809.

35. Merhej D, Ratiney $H$, Diab C, Khalil M, Sdika M, Prost R. Fast multidimensional NMR spectroscopy for sparse spectra. NMR Biomed. 2014;27(6):640-655. doi:10.1002/nbm.3100.

36. Karkouri J, Millioz F, Viallon M, Prost R, Ratiney H. Time samples selection in spiral acquisition for sparse magnetic resonanace spectroscopic imaging. In: ; 2017. https://www2.securecms.com/ICIP2017/Papers/ViewPapers.asp?PaperNum=2064. Accessed October 4, 2017.

37. Coum A, Noury F, Bannier E, Begriche K, Fromenty B, Gandon Y, Saint-Jalmes H, Gambarota G. The effect of water suppression on the hepatic lipid quantification, as assessed by the LCModel, in a preclinical and clinical scenario. Magn Reson Mater Phys Biol Med. 2016;29(1):29-37. doi:10.1007/s10334-015-0508-1. 
Figures

Table 1: Knowledge of the theoretical relative amplitude of the resonance associated with the chemical structure of a typical triglyceride can be either injected in the model $\left(\mathrm{M}_{\text {TG }}\right.$ param $)$ or be used a posteriori for fat composition assessment $\left(\mathrm{M}_{\text {peak }}\right) \cdot \mathbf{f}_{\mathbf{0}_{\mathrm{k}}}$ chemical shift of each resonance $\mathbf{k}$; $n d b$ number of double bonds; $n$ midb number of methylene-interrupted double bonds; $\mathrm{CL}$ chain length (6).

\begin{tabular}{|c|c|c|c|c|}
\hline & & \multicolumn{3}{|c|}{ Parameters conditioning the fat spectrum models } \\
\hline & & & $\begin{array}{c}\mathrm{M}_{\mathrm{TG} \_ \text {param }} \\
\left(\mathrm{Aw}, A f, n d b, n m i d b, T 2^{*}\right)\end{array}$ & $\begin{array}{c}\mathrm{M}_{\text {peak }} \\
\left(\alpha_{1}, \ldots, \alpha_{9}, c_{1}, \ldots, c_{9}\right)\end{array}$ \\
\hline K & & $\boldsymbol{f}_{\mathbf{0}_{\boldsymbol{k}}}(\mathrm{ppm})$ & $n_{k}(n d b, n m i d b, C L)$ & $c_{k}$ \\
\hline 1 & $-\mathrm{CH}=\mathrm{CH}-$ & 5.29 & $2 * n d b+1$ & $A_{f} *(2 * n d b+1)$ \\
\hline 2 & $-\mathrm{CH}_{2}-\mathrm{COO}$ & 4.2 & 2 & $A_{f} * 2$ \\
\hline $2 b$ & $-\mathrm{CH}_{2}-\mathrm{COO}$ & 4.3 & 2 & $A_{f} * 2$ \\
\hline 3 & $\begin{array}{l}-\mathrm{CH}=\mathrm{CH}-\mathrm{CH}_{2}- \\
\mathrm{CH}=\mathrm{CH}-\end{array}$ & 2.75 & $2 * n m i d b$ & $A_{f} * 2 * n m i d b$ \\
\hline 4 & $-\mathrm{CH}_{2}-\mathrm{CH}_{2}-\mathrm{CO}$ & 2.24 & 6 & $A_{f} * 6$ \\
\hline 5 & $\begin{array}{l}-\mathrm{CH}=\mathrm{CH}-\mathrm{CH}_{2-}^{-} \\
\mathrm{CH}_{2^{-}}\end{array}$ & 2.02 & $4^{*}(n d b-n m i d b)$ & $A_{f} * 4^{*}(n d b-n m i d b)$ \\
\hline 6 & $-\mathrm{CH}_{2}-\mathrm{CH}_{2}-\mathrm{CO}$ & 1.6 & 6 & $A_{f} * 6$ \\
\hline 7 & $-\left(\mathrm{CH}_{2}\right)_{n^{-}}$ & 1.3 & $6 *(\mathrm{CL}-4)-8 * n d b+2 * n m i d b$ & $A_{f} *(6 *(\mathrm{CL}-4)-8 * n d b+2 * n m i d b)$ \\
\hline 8 & $-\mathrm{CH}_{3}$ & 0.9 & 9 & $A_{f} * 9$ \\
\hline 9 & $\mathrm{H}_{2} \mathrm{O}$ & 4.7 & $n_{\text {water }}=2$ & $A_{w} * 2$ \\
\hline
\end{tabular}


Figures

Table 2: For in vivo measurements, test-retest variability was calculated. $\mathrm{M}_{\mathrm{TG} \_ \text {param }}$ undersampling with $\mathrm{T} 2$ correction seemed to have the best results with the least variability and estimated values close to the theoretical values. * PUFAindx, MUFAindx and SFAindx values estimated using $\mathrm{ndb}$ and nmidb values. ${ }^{* *}$ in the original paper, the polyunsaturated index (PUI) and unsaturated index (UI) were used and corresponded to $n d b=9 / 2 \times U I$ and $n$ midb $=$ 9/2xPUI

\begin{tabular}{|c|c|c|c|c|c|}
\hline & \multicolumn{5}{|c|}{ Test-retest variability } \\
\hline & $n d b$ & $n m i d b$ & PUFAind $x$ & MUFAindx & SFAind $x$ \\
\hline 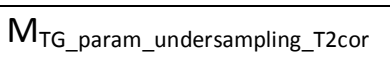 & $2.0 \%$ & $4.1 \%$ & $4.1 \%$ & $0.9 \%$ & $3.6 \%$ \\
\hline $\begin{array}{l}\mathrm{M}_{\mathrm{TG} \_p a r a m} \text { undersampling_T2cor } \\
\text { (without constraints on ndb - } \\
\text { nmidb relationship) }\end{array}$ & $7.3 \%$ & $22.3 \%$ & $22.3 \%$ & $8.7 \%$ & $16.8 \%$ \\
\hline$M_{\text {peak }}$ & $12.5 \%$ & $98.1 \%$ & $98.1 \%$ & $11.6 \%$ & $12.7 \%$ \\
\hline LCModel & $2.7 \%$ & $7.6 \%$ & $7.6 \%$ & $7.8 \%$ & $3.0 \%$ \\
\hline & \multicolumn{5}{|c|}{ Mean \pm SD estimated values } \\
\hline $\begin{array}{l}\text { Values from the } \\
\text { literature }\end{array}$ & & & & & \\
\hline Gas chromatography & $n d b$ & nmidb & PUFA & MUFA & SFA \\
\hline Garaulet et al. (24) & 2.81 & 0.63 & 18.0 & 54.6 & 27.4 \\
\hline Hodson et al. (27) & 2.60 & 0.50 & 16.5 & 54.0 & 29.5 \\
\hline Field et al. (28) & 2.76 & 0.45 & $14.4 \pm 2.7$ & $57.5 \pm 3.1$ & $26.0 \pm 3.0$ \\
\hline$\underline{M R S}$ & $n d b$ & $n m i d b$ & PUFAind $x$ & MUFAindx & SFAind $x$ \\
\hline Hamilton et al. (7) & $2.83 \pm 0.20$ & $0.74 \pm 0.15$ & $24.6^{*}$ & $54.1^{*}$ & $30.3^{*}$ \\
\hline Machann et al. (8) & $2.67^{* *}$ & $0.49 * *$ & $27^{*}$ & $40 *$ & $33^{*}$ \\
\hline $\mathrm{M}_{\mathrm{TG} \_p a r a m \_u n d e r s a m p l i n g \_T 2 c o r}$ & $2.52 \pm 0.23$ & $0.60 \pm 0.11$ & $19.9 \pm 3.7^{*}$ & $44.3 \pm 0.5^{*}$ & $35.8 \pm 4.0^{*}$ \\
\hline $\begin{array}{l}\mathrm{M}_{\mathrm{TG} \_ \text {param_undersampling_T2cor }} \\
\text { (without constraints on ndb - }\end{array}$ & $2.92 \pm 0.26$ & $0.65 \pm 0.15$ & $21.6 \pm 5.1^{*}$ & $54.2 \pm 8.5^{*}$ & $24.2 \pm 6.8^{*}$ \\
\hline
\end{tabular}


Figures

\begin{tabular}{|l|l|l|l|l|l|}
\hline nmidb relationship) & & & & & \\
\hline$M_{\text {peak }}$ & $1.39 \pm 0.29$ & $0.01 \pm 0.02$ & $0.3 \pm 0.5^{*}$ & $46.2 \pm 9.6^{*}$ & $53.5 \pm 9.6^{*}$ \\
\hline LCModel & $1.58 \pm 0.17$ & $0.35 \pm 0.04$ & $11.6 \pm 1.3^{*}$ & $30.0 \pm 5.6^{*}$ & $58.4 \pm 5.5^{*}$ \\
\hline
\end{tabular}


Figures

Table A1: Definition of PUFAindx, MUFAindx and SFAindx calculations with parameters estimated with the two models studied and their measurement uncertainty calculations.

Definition of PUFAindx, MUFAindx and SFAindx calculations with parameters estimated with the two models studied and their measurement uncertainty calculations. For the $\mathrm{M}_{\text {TG_param }}$ the uncertainties of $n d b$ and $n m i d b$ were given directly by the relative CRLBs; for the $\mathrm{M}_{\text {peak }}$ quantification they were calculated with the ratio of amplitude $\boldsymbol{c}_{\boldsymbol{k}}$.

\begin{tabular}{|c|c|c|c|}
\hline & Calculations & \multicolumn{2}{|c|}{ Measurement uncertainty calculations } \\
\hline \multicolumn{4}{|c|}{$n d b$ and $n m i d b$ calculations for the $\mathrm{M}_{\text {peak }}$ with estimated parameters } \\
\hline$n d b$ & $=\frac{1}{2} *\left(9 * \frac{c_{1}}{c_{8}}-1\right)$ & $\frac{\Delta n d b}{n d b}$ & $=\frac{\Delta c_{1}}{c_{1}}+\frac{\Delta c_{8}}{c_{8}}$ \\
\hline$n m i d b$ & $=\frac{9}{2} * \frac{c_{3}}{c_{8}}$ & $\frac{\Delta n m i d b}{n m i d b}$ & $=\frac{\Delta c_{3}}{c_{3}}+\frac{\Delta c_{8}}{c_{8}}$ \\
\hline \multicolumn{4}{|c|}{ PUFAindx, MUFAind $x$ and SFAind $x$ calculations for the two models } \\
\hline PUFAind $x$ & $=100 * \frac{n m i d b}{3}$ & $\frac{\Delta P U F A i n d x}{P U F A i n d x}$ & $=\frac{\Delta n m i d b}{n m i d b}$ \\
\hline MUFAindx & $=100 *\left(\frac{n d b-n m i d b}{3}-\frac{n m i d b}{3}\right)$ & $\frac{\Delta \text { MUFAind } x}{\text { MUFAind } x}$ & $=\frac{\Delta n d b+2 * \Delta n m i d b}{n d b-2 * n m i d b}$ \\
\hline SFAindx & $=100 *\left(1-\frac{n d b-n m i d b}{3}\right)$ & $\frac{\Delta \text { SFAind } x}{\text { SFAind } x}$ & $=\frac{\Delta n d b+\Delta n m i d b}{n d b-n m i d b}$ \\
\hline
\end{tabular}


Table A2: Results of measurement uncertainty calculations of $n d b$, nmidb PUFAindx, MUFAindx and SFAindx with in vivo parameters correspond to the expected fatty acid composition of human subcutaneous abdominal adipose tissue (18\% PUFA, 54.6\% MUFA and 27.4\% SFA (23). The condition number of $J$ explodes for $M_{\text {peak. }}$ For $M_{T G \_p a r a m}$ undersampling reduces the condition number of J for "in vitro". Uncertainties were good for each model but increased when J was poorly conditioned.

\begin{tabular}{|c|c|c|c|c|c|c|c|c|}
\hline Spectrum & Vector of parameters $\theta$ & Sampling & $\begin{array}{l}\text { Condition } \\
\text { number of the } \\
\text { Jacobian matrix }\end{array}$ & $\frac{\Delta n d b}{n d b}$ in $\%$ & $\frac{\Delta n m i d b}{n m i d b}$ in $\%$ & $\begin{array}{l}\frac{\triangle P U F A i n d x}{P U F A i n d x} \\
\text { in } \%\end{array}$ & $\begin{array}{l}\frac{\triangle M U F A i n d x}{\text { MUFAind } x} \\
\text { in } \%\end{array}$ & $\begin{array}{l}\frac{\Delta \text { SFAind } x}{S F A i n d x} \\
\text { in } \%\end{array}$ \\
\hline \multirow{7}{*}{$\begin{array}{l}\text { In vivo } \\
\text { Aw }=1.0 * 1 \mathrm{E}-6 ; \\
\mathrm{Af}=3.7^{*} 1 \mathrm{E}-5 ; \\
n d b=2.7 ; \\
n m i d b=0.54 ; \\
\mathrm{T} 2 *=22.4 \mathrm{~ms} \\
\text { SNR }=210\end{array}$} & $\left(\mathrm{Aw}, A f, n d b, n m i d b, T 2^{*}\right)$ & Full & 91.58 & 0.99 & 6.54 & 6.54 & 6.02 & 7.40 \\
\hline & $\left(\mathrm{Aw}, A f, n d b, n m i d b, T 2^{*}\right)$ & Under & 121.40 & 1.52 & 10.15 & 10.15 & 9.30 & 11.41 \\
\hline & $\begin{array}{l}\left(\mathrm{Aw}, A f, n d b, n \operatorname{mid} b, T 2^{*}\right) \\
\text { with sub-process }\end{array}$ & Full & 33.00 & 0.90 & 1.61 & 1.61 & 2.57 & 3.92 \\
\hline & $\begin{array}{l}\left(\mathrm{Aw}, A f, n d b, n \operatorname{mid} b, T 2^{*}\right) \\
\text { with sub-process }\end{array}$ & Under & 33.99 & 1.35 & 2.45 & 2.45 & 3.89 & 5.92 \\
\hline & $\left(\alpha_{1}, \ldots, \alpha_{9}, c_{1}, \ldots, c_{9}\right)$ & Full & $2.18 \mathrm{E}+03$ & 3.85 & 14.90 & 14.90 & 16.34 & 21.94 \\
\hline & $\left(\alpha_{1}, \ldots, \alpha_{9}, c_{1}, \ldots, c_{9}, f_{1}, \ldots, f_{9}\right)$ & Full & $1.13 E+04$ & 4.89 & 18.98 & 18.98 & 20.80 & 27.91 \\
\hline & $\left(\alpha_{1}, \ldots, \alpha_{9}, c_{1}, \ldots, c_{9}, \beta_{1}, \ldots, \beta_{9}, f_{1}, \ldots, f_{9}\right)$ & Full & $1.22 \mathrm{E}+06$ & 8.51 & 34.20 & 34.20 & 36.98 & 49.33 \\
\hline
\end{tabular}


Figures

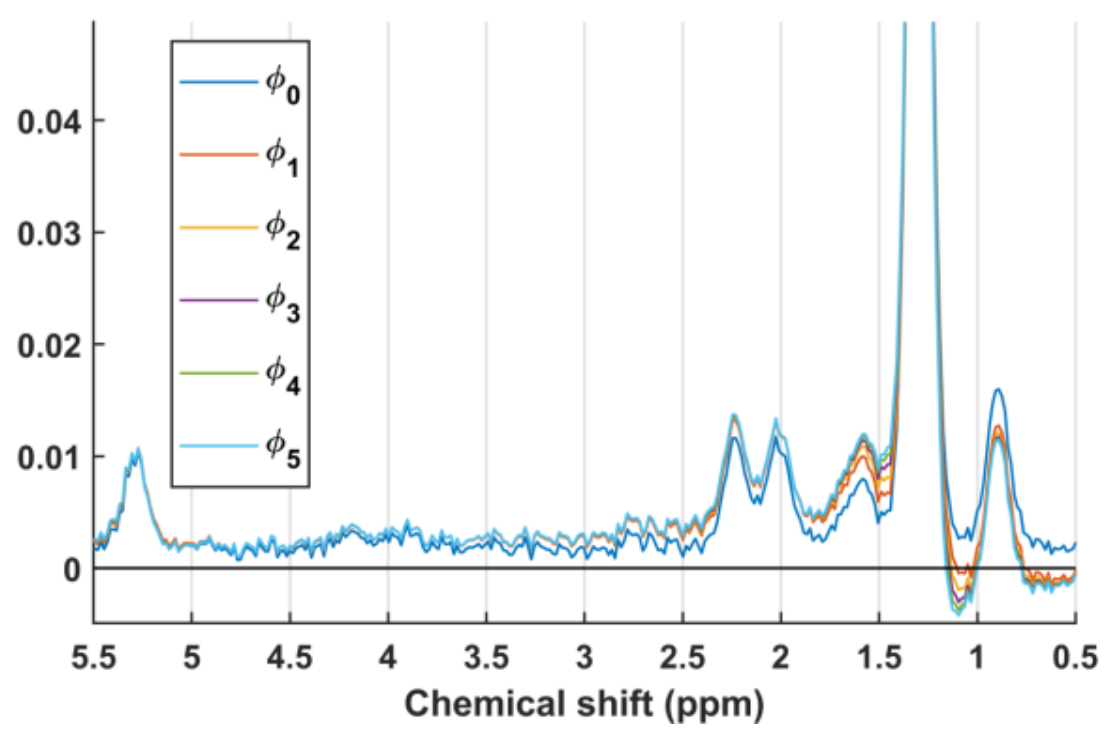

Figure 1 : Simulated adipose tissue lipid MR spectra used in the Monte Carlo studies, with different degrees of baseline distortion, simulating the eddy current effect; $S N R=210$. The phase variation was simulated using the following exponential model: $\phi_{i}(t)=\exp \left(-\frac{t}{\tau_{i}}\right), \tau_{i}=\frac{50+50 * i}{3} m s$ for $0 \leq \mathrm{t} \leq 100 \mathrm{~ms}$, with $\mathrm{i}=0 . .5$, for the 6 spectra above. 


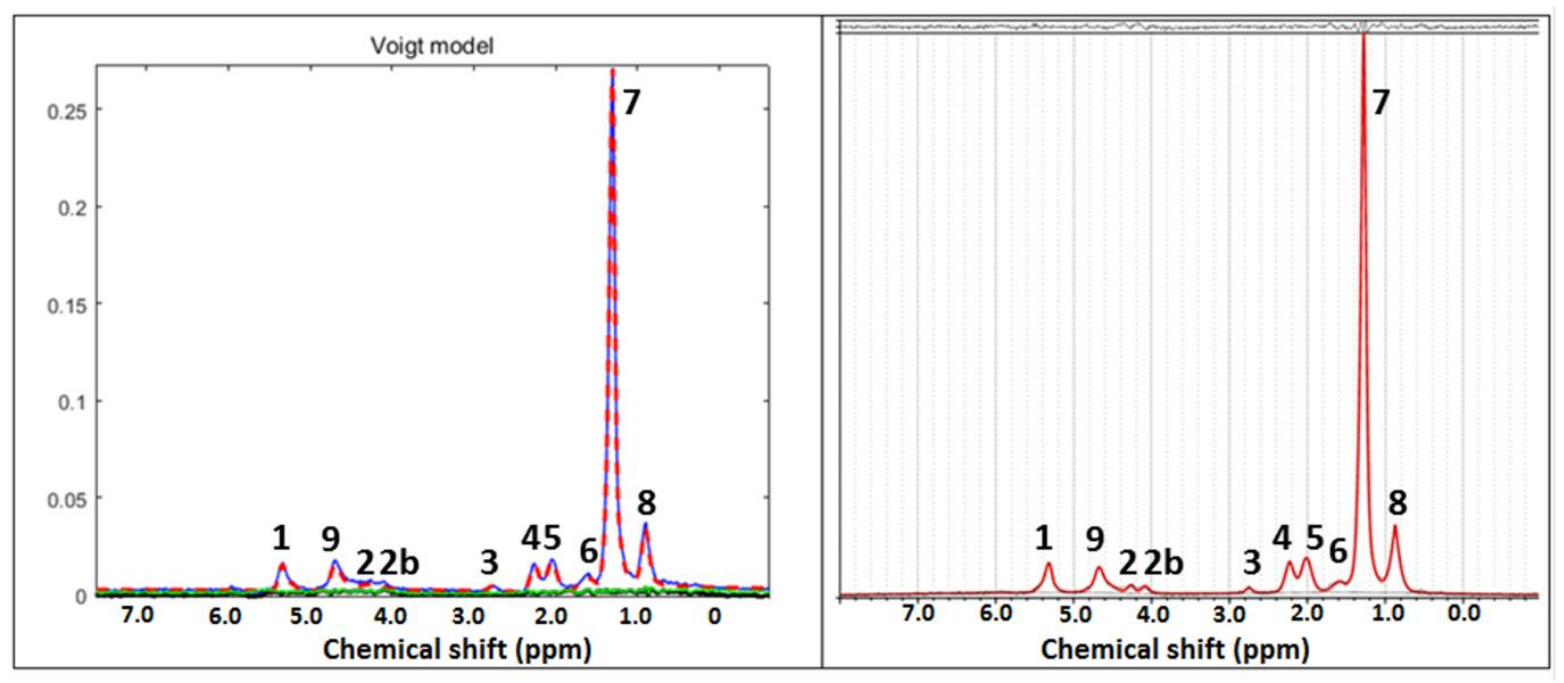

Figure 2: In vivo MRS was fitted with two models: Mpeak (Voigt model) on the left and LCModel on the right. For botmodels, the fitted curve is represented by a red line. For the left spectrum the original spectrum is represented with a blue line, the residual is a black line and the absolute residual is a green line on the same window. For the right spectrum, the residual is represented with a black line in a separate window just above the spectrum. Different peaks are noted as well: 1 , olefinic $(-\mathrm{CH}=\mathrm{CH}-) ; 2$ and $2 \mathrm{~b}$, glycerol $(-\mathrm{CH} 2-\mathrm{O}-\mathrm{CO}-)$; 3 , dyacil $(-\mathrm{C}=\mathrm{C}-\mathrm{CH} 2-\mathrm{C}=\mathrm{C}-) ; 4, \alpha$-carboxyl $(-\mathrm{CO}-\mathrm{CH} 2-$ $\mathrm{CH} 2-) ; 5, \alpha$-olefinic (-CH2-CH=CH-CH2-); $6, \beta$-carboxyl (-CO-CH2-CH2-); 7, methylene (-CH2-); 8, methyl (-CH3). For this example, even if the residual is very small the fatty acid compositions found with the two models are clearly different (3.8\% PUFA, 43\% MUFA and 53.6\% SFA with the Voigt model and 9.6\% PUFA, 50\% MUFA and 40.5\% SFA with LCModel). 


\section{Simulated signals with the same T2 for each peak}
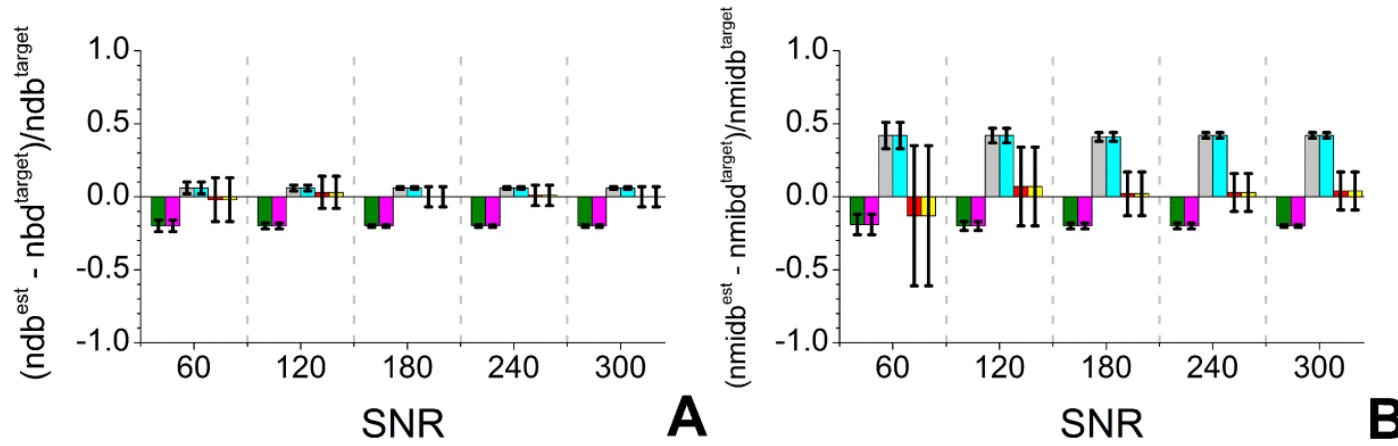

Simulated signals with different T2s for each peak
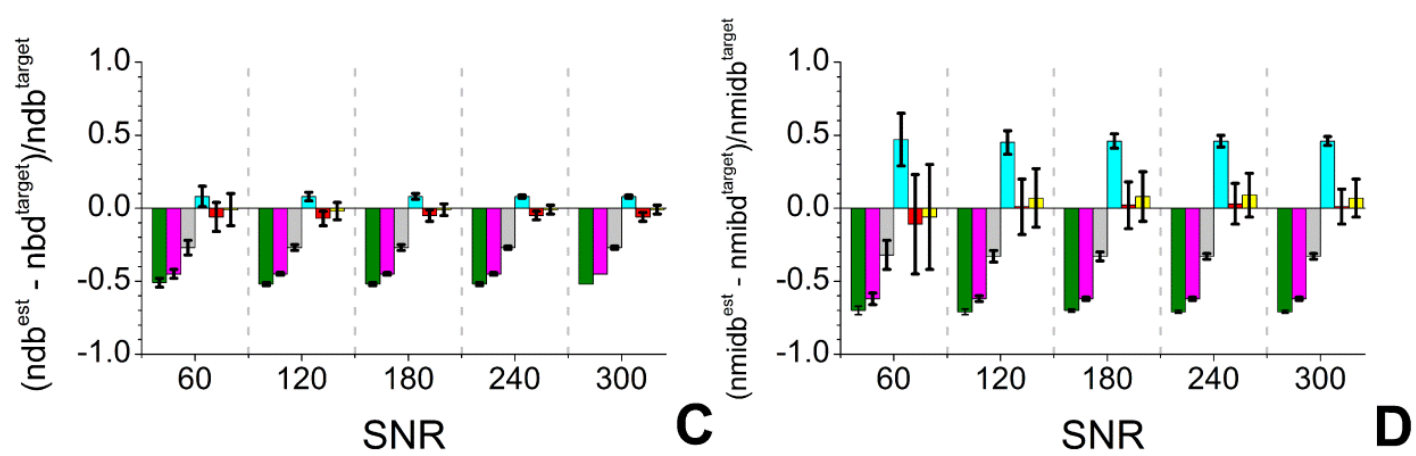

Simulated signals with phase distortion
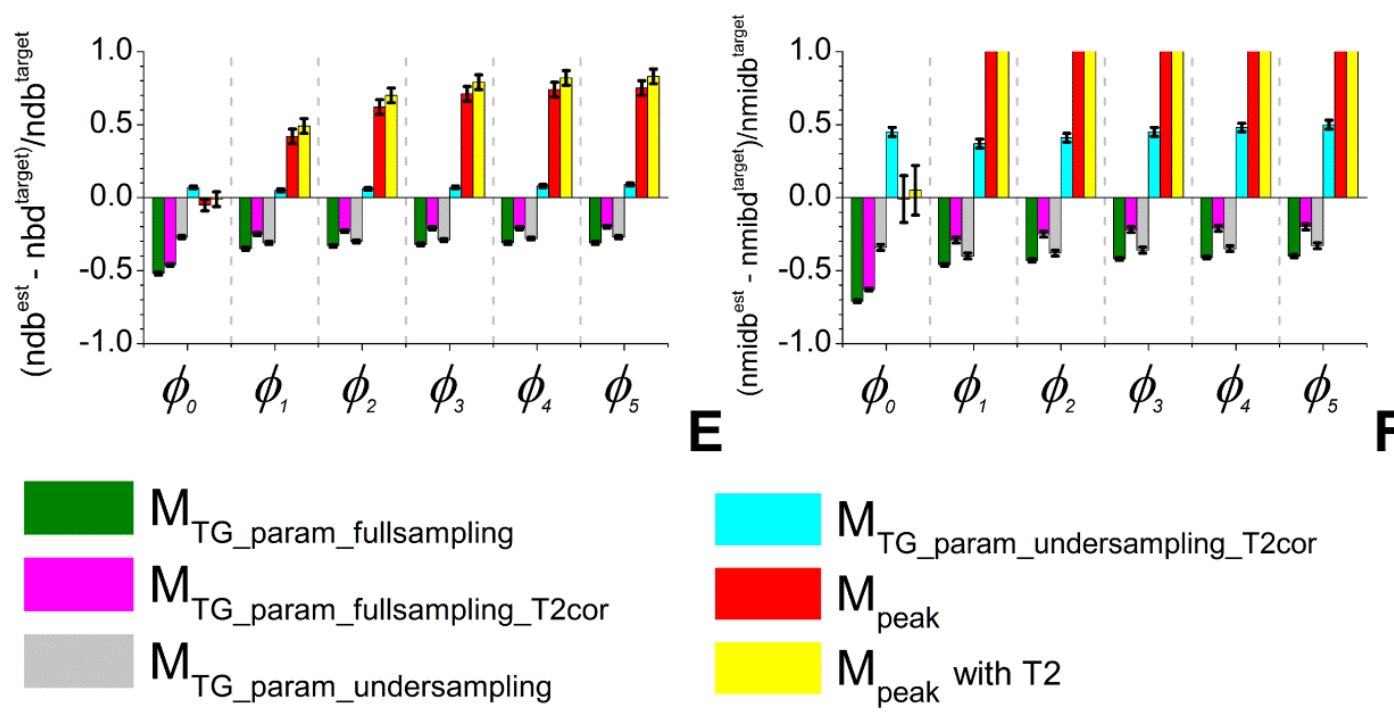

Figure 3 : Monte Carlo simulation results. The simulated signal was composed with $n d b^{\text {target }}=2.7$ and $n m i d b^{\text {target }}=0.54$, and a PDFF of $97 \%$. These parameters should model a spectrum close to the in vivo spectrum. Bar plots showing the mean \pm SD of the difference as a percentage between the estimated value (est) by different quantification methods and the target value (target) of $n d b(A, C, E)$ or $n$ midb $(B, D, F)$. The results are obtained from 100 random draws of noise added to simulated signals with the same T2 for each peak (A, B), with different T2s for each peak (C, D) with a phase distortion (E, F). 
Figures
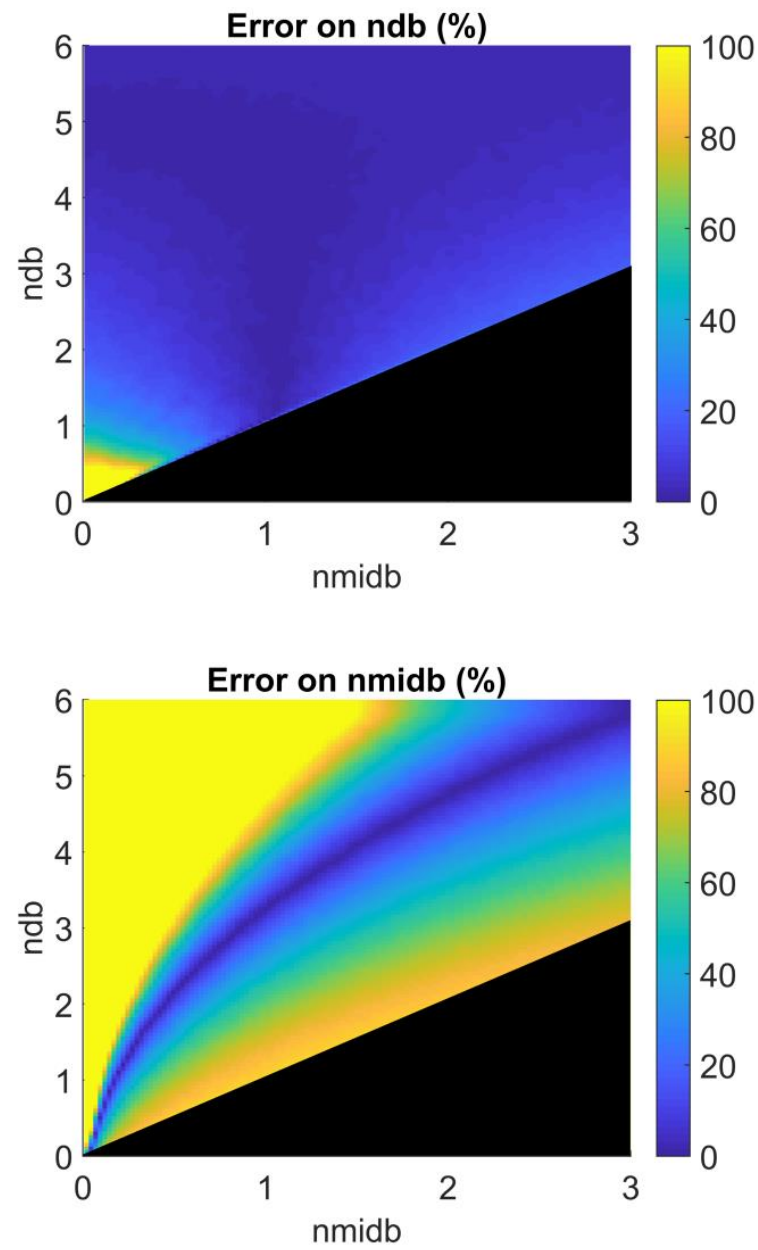

Figure 4 : Absolute quantification errors on $n d b$ and $n m i d b$ according to the FA composition for the $\mathbf{M}_{\text {TG_param_undersampling_T2 cor }}$ with undersampling and T2 correction. The black area has no physical meaning $(n m i d b>n d b)$ and has not been evaluated. The error varies according to FA composition. The part with nmidb varying from 0.30 to 0.80 and ndb varying from 1.90 to 2.80 correspond to the excepted in vivo values. 
Figures

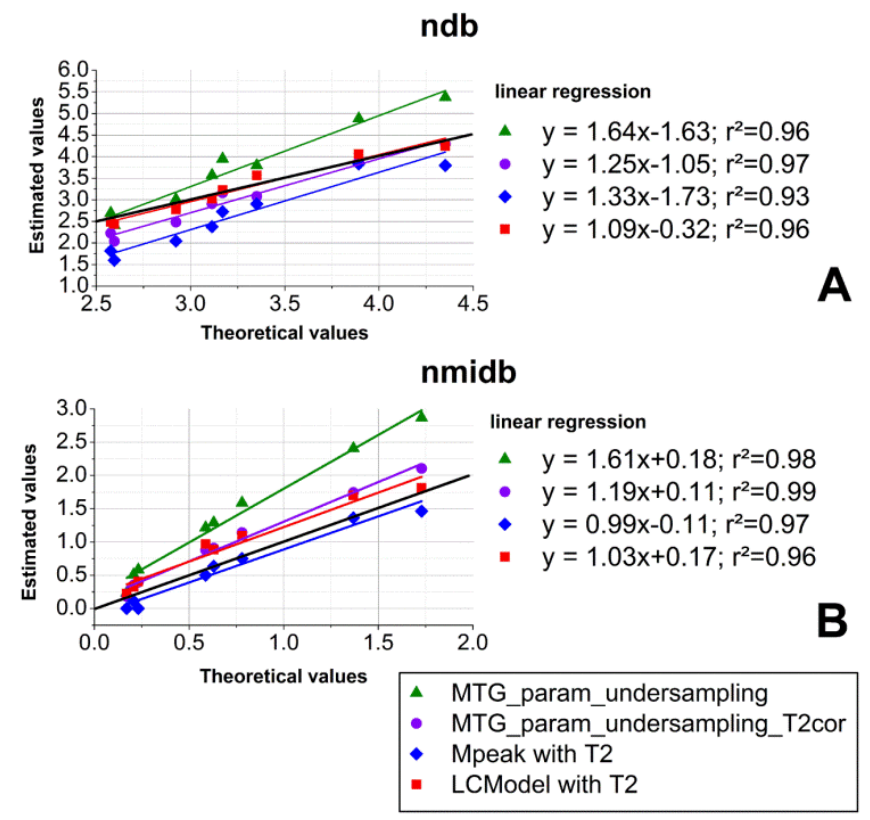

Figure 5 : Estimated values as a function of theoretical values for $n d b$ (A) and $n m i d b$ (B) from in vitro MRS acquired with a PRESS at 4.7T on eight vegetable oils (avocado, canola, hazelnut, walnut, pistachio, grape-seed, sesame and olive).

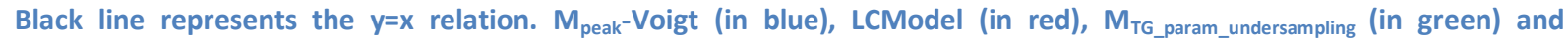

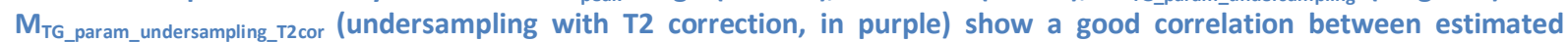
values and theoretical values. LCModel estimated $n d b$ values in closer agreement with the theoretical values. Voigt model gave the best estimation of $n m i d b$ but underestimated $n d b$ values. LCModel and $\mathbf{M}_{\text {TG_param_undersampling_T2cor }}$ gave consistent values of $n d b$ and $n m i d b$. 